\title{
La importancia de la información documentada. Caso de actualización documental de un Sistema de Gestión de la Calidad para certificación bajo la norma ISO 9001:2015
}

\begin{abstract}
Ángel Monzalvo Hernández ${ }^{a}$, Heriberto Niccolas Morales ${ }^{b}$, Germán Reséndiz López ${ }^{a}$, Jaime Garnica González ${ }^{b}$, Noel Iván Toto Arellano ${ }^{a}$, Heriberto Canales Gutierrez ${ }^{\mathbf{c}}$, ${ }^{a}$ Universidad Tecnológica de Tulancingo, Tulancingo, México - angelmonzalvo@utec-tgo.edu.mx,

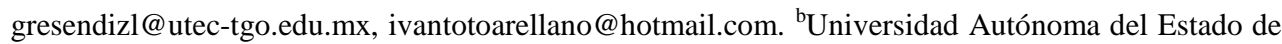
Hidalgo, Mineral de la Reforma, México - hnicolas@uaeh.edu.mx, jgarnica@uaeh.edu.mx. ${ }^{\text {TTHSA }}$ EM S.A. DE C.V., Tulancingo, México - hcanales@tihsakomac.com
\end{abstract}

\section{Resumen}

La documentación de una empresa representa su capital intelectual, ahí se plasma el "saber hacer" que se ha adquirido con la experiencia y el paso de los años. Si no se preserva adecuadamente ese conocimiento se podría perder y provocaría retrabajos y deficiencia en las operaciones. El objetivo de este trabajo es compartir el resultado de un proyecto de intervención en una empresa para lograr la transición de la norma ISO 9001:2008 a la ISO 9001:2015, desarrollando una a una las cláusulas y requisitos solicitados para lograr la certificación.

Se describe cómo se desarrolló el sistema nombrado "Matriz de los procesos" el cual incluye de manera completa los requisitos en orden solicitados por la norma, así como los documentos, archivos y formatos asociados. Se elaboró una matriz de procesos que incluye información anterior proporcionada por el antiguo sistema nombrado "Matriz de correlación" la cual cumplía con la norma ISO 9001:2008. La matriz de procesos se desarrolló con el objetivo de identificar de manera rápida y sencilla que documentación de la empresa cumple con determinados puntos de la norma. Además, esta incluye de manera breve y concisa las entradas correspondientes en los procedimientos de la empresa y las salidas que se generan de dichos procedimientos. La transición de la norma ISO versión 2008 a 2015 generó que se debiera crear nueva información y realizar modificaciones y/o actualizaciones a documentos e información con la que la empresa ya contaba, dichos cambios se muestran explicando las etapas de cambio de estos y las consideraciones o recomendaciones propuestas por la norma. Se diseñó un mapa general de procesos con ayuda de hoja de 
cálculo (Excel), tomando como base el ciclo PHVA (Planear, Hacer, Verificar, Actuar) incluido en la norma ISO 9001:2015.

Palabras clave: Documentación, Gestión de la Calidad, ISO 9001:2015, Matriz de procesos, Organización.

\section{Introducción}

Hoy en día las nuevas necesidades y requisitos de los clientes obligan a las empresas a realizar cambios en sus sistemas y estructura de organización para poder satisfacerlos. Sin embargo, esto no es algo que deba preocupar, al contrario, dichos cambios y actualizaciones generan numerosos beneficios a las empresas que van desde aumentar la calidad de los productos o servicios que ofrece hasta aumentar la competitividad y la cantidad de clientes. La calidad ha sido un tema de interés creciente para las empresas y clientes desde hace más de 30 años. La transición de la norma ISO 9001 posee varios beneficios entre los cuales destaca la integración de otras normas de la familia ISO, un enfoque hacia el ahorro económico de la empresa, formas más efectivas de evaluar la eficacia de los cambios que se llevan a cabo en la empresa y proporcionar un mejor estatus, ya que al poseer una certificación en ISO 9001, se genera una mayor confianza tanto en clientes como en proveedores (Organización Internacional de Normalización, 2015b).

Actualmente existen normas ISO que regulan desde procedimientos hasta productos a nivel nacional e internacional. El objetivo de estas normas es controlar y mejorar la calidad y procesos de producción de las empresas, es por ello que las empresas buscan lograr estas certificaciones, ya que les posibilita ampliar su mercado y prepararse para ser más competitivos (Miletić, Jovanović, Jovanović y Buha, 2017).

En este contexto, este trabajo presenta una breve descripción del proceso de transición de la norma ISO 9001:2008 a la ISO 9001:2015, realizado para la empresa TIHSA EM S.A. de C.V., que es una empresa mexicana, ubicada en el Municipio de Tulancingo, estado de Hidalgo. La generación de la nueva norma ISO 9001:2015, provocó que gran parte de la información, documentación, procesos y sistema llamado "Matriz de correlación ISO" que utilizaba la empresa fueran obsoletos al estar basados en la norma ISO 9001:2008. Lo anterior, representó una problemática de gestión documental en la empresa, por lo que fue necesario plantear un proceso integral para actualizar la documentación que respondiera a la pregunta: ¿Qué aspectos estratégicos eran necesarios para la transición de la norma ISO 9001: 2015? Se planteó así la necesidad de analizar los factores críticos de operación de la empresa, lo que permitió detectar los cambios por desarrollar tanto en las áreas administrativas como en áreas de producción, que implicaba actualizar o diseñar todos 
aquellos formatos, documentos, archivos y procesos que resultaban obsoletos o que ya no cubrían por completo con los lineamientos demandados por la nueva norma.

El objetivo del trabajo es compartir el resultado de un proyecto de intervención en una empresa para lograr la transición de la norma ISO 9001:2008 a la ISO 9001:2015, desarrollando una a una las cláusulas y requisitos solicitados para lograr la certificación. Como objetivos específicos de la intervención en la empresa se determinaron los siguientes:

- Diseñar la matriz de los procesos

- Elaborar el mapa general de procesos TIHSA EM.

- Participar en la actualización de la planeación estratégica de la empresa. De forma específica en lo relacionado con el sistema de gestión de la calidad.

- Conocer y familiarizarse con el anterior sistema empleado por la empresa.

- Determinar que documentos son útiles y cuáles no para la norma ISO 90012015.

- Descargar al nuevo sistema los documentos útiles para la nueva versión de norma.

- Descargar al nuevo sistema las evidencias y/o información obtenida del desarrollo de las cláusulas de la norma.

\section{Marco de referencia}

\subsection{Antecedentes de la empresa TIHSA EM}

La empresa TIHSA EM S.A. de C.V. surge en el año de 1972. El giro de la empresa pertenece al sector metal-mecánico, ya que es fabricante y maquiladora de partes componentes y productos para la industria automotriz, de construcción y minera, entre sus clientes cuenta con empresas distribuidoras en la República Mexicana en las ciudades de Querétaro, México, Guadalajara, Monterrey, Tamaulipas; Guanajuato y San Luis Potosí, así como en el extranjero. TIHSA EM, ha obtenido tres veces el premio Hidalgo de la calidad, la primera ocasión fue en el año de 1994, seguido por el año 1998 y por último en 2007. También logró la certificación QS-9000 en el año 2004, lo que muestra el fuerte enfoque hacia la calidad que ha tenido la empresa en las últimas tres décadas.

La gestión de la calidad no es un elemento rígido, requiere renovarse constantemente para poder satisfacer las nuevas necesidades de las empresas y de sus clientes. TIHSA EM ha logrado establecer un proceso de planeación estratégica que le ayuda a renovar su Misión, Visión, Objetivos empresariales, Política de Calidad, Filosofía y Valores de una forma 
La importancia de la información documentada. Caso de actualización documental de un Sistema de Gestión de la Calidad para certificación bajo la norma ISO 9001:2015

continua. Este enfoque le ha permitido un desarrollo importante como empresa en la región de Tulancingo, Hidalgo, México.

\subsection{La calidad y los beneficios de la normalización}

La calidad se puede definir como el conjunto de características que posee un producto o servicio y que le otorgan la capacidad de satisfacer las necesidades y expectativas de un cliente (Nava, 2006). De acuerdo a Palafox (2005), citado por Martínez (2007), la calidad supone que el producto o servicio debe cumplir con las funciones y especificaciones para lo que ha sido diseñado y que deberán ajustarse a las expresadas por los consumidores o clientes del mismo.

Las normas son un modelo, patrón o criterio a seguir. Una norma tiene valor de regla y tiene el propósito de establecer las características que debe poseer un objeto y los productos que han de tener una compatibilidad para ser usados a nivel internacional. Por ejemplo, el problema que ocasiona a muchos usuarios los distintos modelos de enchufes que existen a escala internacional para poder acoplar pequeñas máquinas de uso personal tales como secadores de cabello, máquinas de afeitar, cargadores de celular entre otros, cuando se viaja de un país a otro y que provoca el mal funcionamiento del aparato o que no se pueda usar se puede evitar al aplicar una normalización en especificaciones. La incompatibilidad repercute en muchos campos, por lo que la normalización de los productos cobra mayor importancia en un mundo más globalizado.

\subsubsection{Las normas ISO}

Las normas ISO se establecen por el Organismo Internacional de Estandarización (ISO), que es una entidad formada por 81 organismos de normalización de varios países del mundo cuya función principal es editar y evisar normas internacionales para facilitar el intercambio de biens y servicios (Nava, 2005; Organización Internacional de Normalización, 2015b). Las normas se componen de estándares y guías relacionados con sistemas y herramientas específicas de gestión que se aplican en las diferentes empresas productoras de bienes o prestadoras de servicios (Cruz, López y Ruíz, 2017). Las normas ISO son un conjunto de normas orientadas a ordenar la gestión de una empresa en sus distintos ámbitos. La alta competencia internacional acentuada por los procesos globalizadores de la economía y el mercado, así como el poder e importancia que ha ganado la figura y la opinión de los consumidores, propicia que dichas normas, pese a su carácter voluntario, hayan ganado un gran reconocimiento y aceptación internacional.

La finalidad principal de las normas es orientar, coordinar, simplificar y unificar los usos para conseguir menores costos de operación y efectividad. Tienen valor indicativo y de guía y en la actualidad su uso se ha extendido generando gran interés por parte de las 
empresas en seguir las normas existentes, porque desde el punto de vista económico reduce costos, tiempo y trabajo (Retos Supply Chain, 2014). Lo que se pretende lograr es que las normas ISO sean un elemento rector en cuanto a la importancia que tienen las relaciones de confianza entre los clientes y los proveedores (Retos Supply Chain, 2014).

\subsection{Evolución de norma ISO 9001}

En la década de los 80’s del siglo XX, el control de calidad pasó a ser garantía de calidad. La calidad ya no se limitaba únicamente al producto, sino que englobaba todo el proceso y la cadena de producción que debían garantizar la conformidad del producto. Mediciones y pruebas de calidad del producto eran realizadas en la cadena de producción y era deber de la empresa demostrar la calidad del producto al cliente.

Al principio de los años 2000, la garantía de calidad que se realizaba únicamente en las cadenas de producción pasó a ser dirigida, gestionada, y mejorada bajo la forma de un sistema de gestión. El producto, así como los servicios creados y aprovisionados por la empresa pasan a estar bajo la responsabilidad del sistema de gestión. La empresa no solo tenía que garantizar la conformidad de sus productos y de sus servicios, sino que también debía satisfacer al cliente y brindarle la prueba de conformidad.

En el 2015, el sistema de gestión de la calidad se extiende y ya no solo abarca a los clientes, sino que también a toda parte interesada que sea pertinente para la empresa (stakeholders), partes que conformen el ecosistema de la organización. La nueva versión de ISO 9001:2015 promueve una mejor integración del estándar con otras normativas similares, lo que se sitúa en línea con el nuevo concepto de calidad, mucho más amplio e integrado con otros elementos corporativos como: la Gestión de los Riesgos, la Responsabilidad Social o la Gestión Medioambiental. Se gestiona, modifica y mejora el sistema de gestión de la calidad - que se encuentra bajo la responsabilidad de la alta dirección - con la ayuda de riesgos y oportunidades identificadas y juzgadas pertinentes para la empresa, teniendo en cuenta el contexto, los desafíos y a todas las partes interesadas pertinentes para la empresa (Rodríguez, 2017).

En la Figura 1 se muestra un esquema con los siete conceptos básicos que tiene la versión ISO 9001:2015 y que sustentan la "estructura de alto nivel" (Organización Internacional de Normalización, 2015a; Gobierno del Estado de México, 2016; Instituto Mexicano de Normalización y Certificación, s.f.). 
La importancia de la información documentada. Caso de actualización documental de un Sistema de Gestión de la Calidad para certificación bajo la norma ISO 9001:2015

\subsection{La norma IAFT 16949}

En el ámbito de la industria automotriz la International Automotive Task Force (IATF) ha publicado el estándar IATF 16949:2016, el cual ha sustituido a ISO/TS 16949:2009 como referencia para el desarrollo de sistemas de gestión de la calidad en la industria automotriz (Prudencio, 2016). La empresa TIHSA EM requiere ajustarse también en el cumplimiento de esta norma por lo que su sistema de gestión de la calidad debe incluirla.

La norma IATF 16949:2016 ha sido desarrollada con base en la nueva estructura de alto nivel para los sistemas de gestión de ISO que fue publicada en 2012, bajo la cual todas las nuevas normas publicadas a partir de ese año y las normas que se han actualizado tienen la misma estructura, facilitando su implementación de manera integrada y optimizando los recursos e inversión que implica para las organizaciones (Prudencio, 2016).
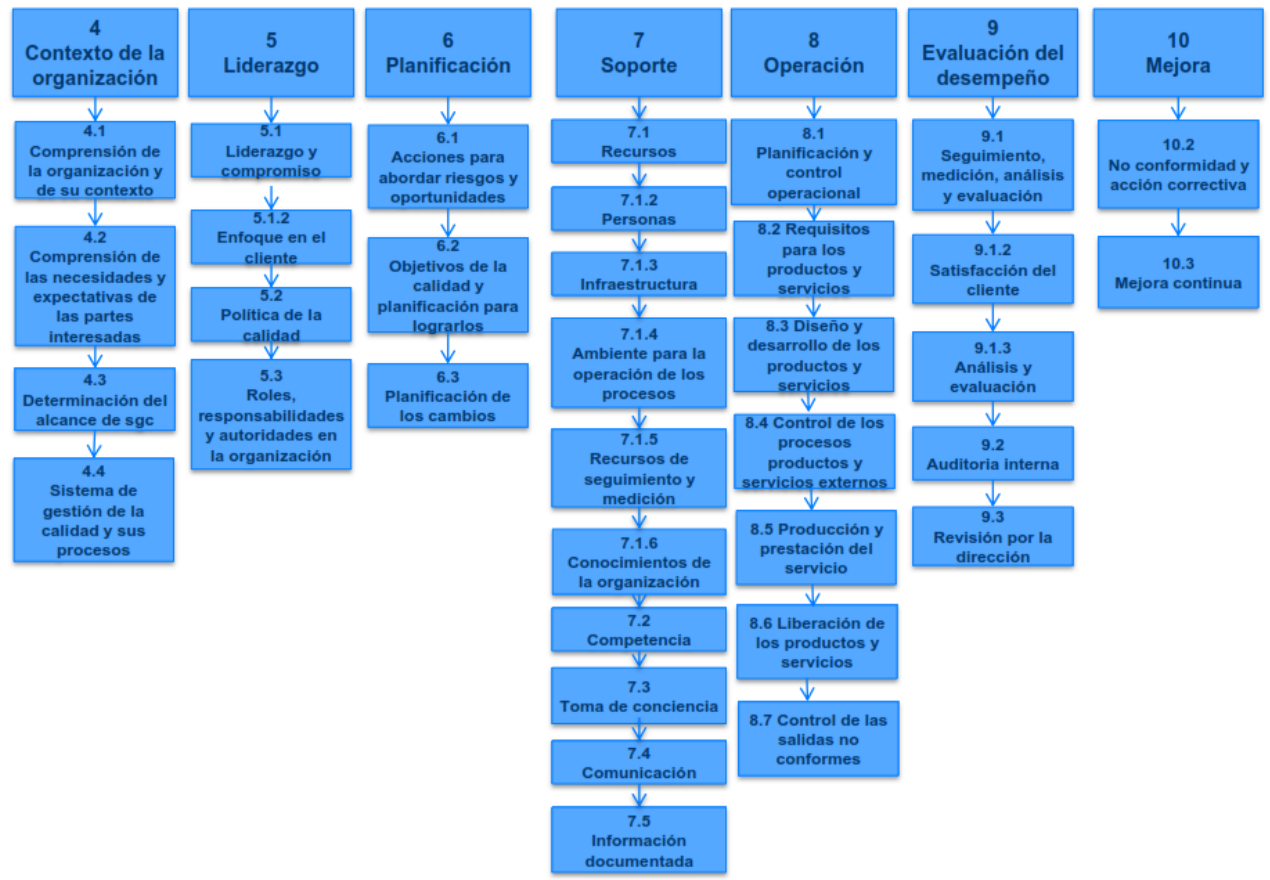

Fig. 1 Siete conceptos básicos de la norma ISO 9001:2015

Fuente: Instituto Mexicano de Normalización y Certificación, A.C. (s.f.) 


\section{Metodología}

Para el desarrollo del trabajo descrito se utilizó el enfoque sistémico, ya que ayuda a representar la secuencia de acontecimientos o actividades relacionadas para lograr un determinado fin. El enfoque sistémico tiene como punto de referencia el concepto de sistema, que es un conjunto de elementos interrelacionados con un objetivo común (Van Gigch, 2006).

En la Figura 2 se muestra un modelo de enlaces de cadena basado en Marquis (1969) para representar el esquema metodológico seguido en el presente trabajo.

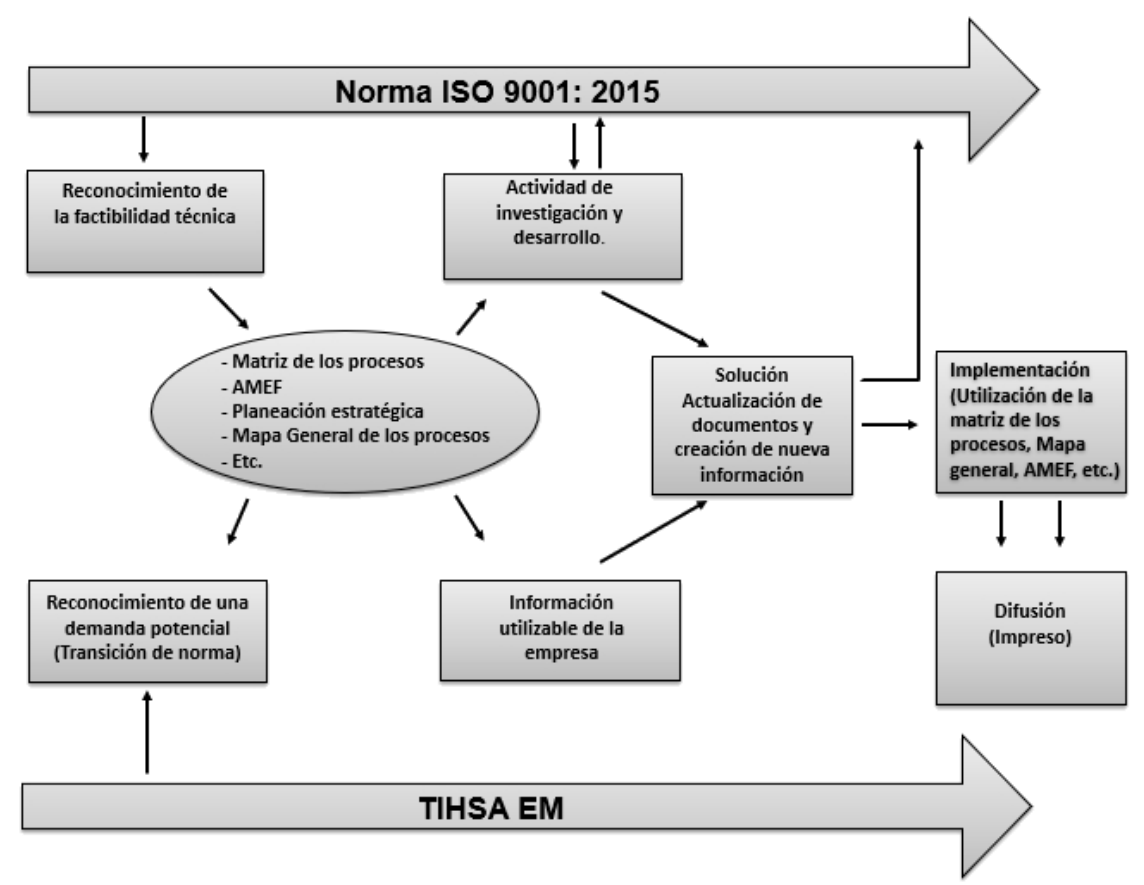

Fig. 2 Modelo de enlaces para la intervención en la empresa TIHSA EM con enfoque sistémico Fuente: Elaboración propia con base en Marquis (1969).

El periodo de realización del proyecto comprendió de julio de 2017 a noviembre de 2018. Las actividades realizadas como parte de la metodología de trabajo se indican a continuación: 
1. Conocer y explorar el anterior sistema empleado para familiarizarse y realizar propuestas de sistema en el cual se incluyan los apartados correspondientes a los solicitados por la norma ISO 9001:2015.

2. Elaboración del mapa general de procesos TIHSA EM.

3. Descargar la información proporcionada por la norma ISO 9001:2015 y la norma IATF 16949 en el nuevo modelo de sistema "Matriz de los procesos" para dar una estructura, orden y seguimiento. Además, incluir la información, resultados y actividades obtenidos por el trabajo con el punto no. 4 de la misma.

4. Analizar y evaluar los documentos incluidos en el anterior sistema para determinar si aún son funcionales o no.

5. Seguimiento al sistema realizado. Asignación de nuevos códigos a los documentos y formatos.

6. Descargar al sistema la información y documentos obtenidos del avance y desarrollo de las cláusulas por parte de la norma ISO 90012015 y la norma IATF 16949.

7. Elaborar el informe final para su entrega y revisión del asesor empresarial y académico. Hacer las modificaciones correspondientes hasta lograr la autorización del reporte final.

\section{Resultados}

La transición que se realizó respecto a la norma ISO 9001:2008 a la 9001:2015, presentó un gran reto tanto para el personal administrativo como para los trabajadores y operarios. Los documentos identificados en la matriz de correlación enmarcada en la ISO 9001:2008 fueron 23 relativos a políticas, 56 de procedimientos, 22 instructivos, 3 manuales y 126 formatos. Los cambios implicaron modificaciones de gran alcance y otras de menor alcance en diferentes secciones de la empresa que abarcan desde la administración hasta la producción. Se generó una Matriz de Procesos que considera un mapeo de procesos (entradas y salidas) bajo la ISO 9001:2015. Tomando como base el mapa de procesos y el ciclo PHVA se dio inicio a la elaboración del mapa general de procesos de TIHSA. Dicho mapa debía contener de manera breve y concisa los procesos y sectores / áreas a los que pertenecen. La intervención realizada en TIHSA dio lugar a la propuesta de una "Matriz de alineación", que se realizó para incluir en la planeación estratégica de la empresa, la alineación de la visión, misión, Sistema de Gestión de Calidad, política de calidad y objetivos de la empresa, con la finalidad de poder evaluar que enfoque dan a características que poseen o que deberían de cubrir en común. Dichas características abarcan los clientes, 
procesos productivos, industrias, productos, materiales, maquinaria y equipo, mano de obra y certificaciones.

Al continuar con el desarrollo de las cláusulas correspondientes a la ISO 9001:2015, se detectó que TIHSA EM no contaba con una lista de riesgos a considerar y con los que se debiera trabajar. Para poder cubrir las necesidades y requisitos que se solicitaban se creó un listado de riesgos el cuál posteriormente se trasladó a un diagrama de Ishikawa con el objetivo de identificar los riesgos críticos que eran más propensos a suceder, esto con el objetivo de trabajar con los mismos para poder controlarlos y de ser posible, eliminarlos. También se realizó una revisión de la descripción de los puestos de trabajo basados únicamente en las actividades que afectarían directamente la estructura o producción de la organización. Posteriormente, se procedió a asignar los nuevos códigos a los documentos y/o archivos que ya estaban listos o los que se identificaron de manera correcta dentro de la matriz de procesos, esto con la finalidad de poder identificarlos basados en las características y especificaciones de la nueva norma. Para la asignación de nuevos códigos se hizo uso de uno de los instructivos que se actualizó, el IN-7.5.2-01-R2-100318 ya que este es el que determina la manera correcta de asignar un código a un nuevo archivo o un archivo modificado.

La certificación del Sistema de Gestión de la Calidad se obtuvo en noviembre de 2018 por parte del organismo certificador American Trust Register, S.C. (ATR) con número de certificado 2496, al cumplir satisfactoriamente con lo requerido por el organismo. En el sistema creado, la matriz de los procesos, es la base estructural la cuál contiene gran parte de la información relevante de la empresa. Se realizaron cambios a información relevante de la empresa tal como la planeación estratégica que incluye la razón de ser, objetivos, políticas y organigrama, entre otros. También se realizaron propuestas de cambios a archivos que pudieran ser de ayuda en la justificación de documentos o en la creación de nueva información. La matriz creada ha resultado de mucha utilidad ya que permite identificar de manera clara y concisa los requisitos de la norma y los documentos que posee la empresa que cumplen con dichos requisitos. Anteriormente se contaba con un sistema llamado matriz de correlación, sin embargo, carecía de estructura y generaba algunas confusiones al tratar de saber con qué puntos específicos estaba cumpliendo cada documento contenido en ella.

\section{Consideraciones finales}

La realización del proyecto de intervención en la empresa TIHSA EM surgió de la necesidad de actualizar su sistema de calidad para alcanzar la certificación en la norma 
ISO versión 9001:2015. El enfoque de la nueva norma ISO 9001:2015 se basa en la gestión del riesgos, el Sistema de Gestión de Calidad (SGC) y la estructura funcional de la empresa, poniéndose especial énfasis en la interconexión y las relaciones existentes entre estos tres elementos. A partir del estudio de los cambios de la norma, se encontró que el análisis de riesgos, con una vocación preventiva, pasa a tener una importancia crítica en la filosofía de ISO 9001:2015. Se observó que ahora se pone el énfasis en la necesidad de identificar los riesgos para poder eliminarlos o minimizar sus consecuencias a través de la puesta en marcha de procesos de mejora continua del sistema.

Para lograr esta nueva certificación fue necesario realizar numerosos cambios al sistema de la empresa, que abarcó a la información, documentos y procesos, con la finalidad de cubrir las especificaciones que la norma solicitaba. El principal desafío que se presentó fue familiarizarse con el anterior sistema e identificar cada uno de los archivos, documentos y formatos que este contenía para así poder analizarlos y determinar si cumplían o no a las demandas solicitadas por la norma ISO 9001:2015. Fue necesario crear algunos nuevos archivos que las cláusulas de la norma solicitaban y para los cuales la empresa no contaba con ellos. Otros más como el mapa general de los procesos necesitaron una actualización obligatoria ya que al estar realizados en base a especificaciones pasadas, con la nueva versión quedaban obsoletos. La evaluación y análisis de documentos requirió de una gran cantidad de tiempo y el determinar los cambios o modificaciones a realizar se hizo con ayuda de un asesor académico.

Respecto a la matriz de los procesos y los documentos que contiene, la identificación de dichos documentos se puede realizar ahora de manera clara y sencilla. Con el nuevo sistema creado es mucho más fácil identificar qué documento cubre un punto específico de la norma, ya que se agregaron las cláusulas completas de esta para evitar tener que acceder a otro documento que especificara que es lo que se debía de cumplir. Se construyeron las bases y estructura general del sistema que serviría de apoyo para la certificación. En realación al sistema Matriz de los procesos, este contiene las entradas y salidas de los procesos correspondientes a las cláusulas de las normas. A las entradas y salidas se les mapeo, para generalizar las actividades que se realizaban. De igual manera, el mapeo de los procesos ayudó a identificar de forma más rápida los archivos y/o documentos que deben encontrarse para cubrir con determinadas cláusulas. Lo anterior, ayudará a que los documentos y archivos que se identificaron y añadieron se encuentren en constante actualización y modificación con la finalidad de volverlos útiles para las certificaciones correspondientes.

Finalmente, se recomienda analizar constantemente las oportunidades presentes y futuras para tomar acciones con la finalidad de mejorar los procedimientos dentro de la empresa. También es recomendable mejorar la comunicación y flujo de información dentro de la empresa para aumentar la respuesta ante situaciones no deseadas que pudiesen complicar 
el ritmo de producción o la integridad de la empresa. Revisar y actualizar la información de la empresa es muy importante ya que se encontraron datos y archivos obsoletos que no generaban ningún aporte y que en cambio ocasionaban confusiones en la identificación de archivos e información que si era útil y de valor.

\section{Referencias}

Cruz, F. L., López, A. P. y Ruíz, C. (2017). Sistema de gestión ISO 9001-2015. Técnicas y herramientas de ingeniería de calidad para su implementación. Revista Ingeniería, Investigación y Desarrollo. 17(1), 59-69.

Gobierno del Estado de México. (2016). Guía de apoyo para la transición de Sistemas de Gestión de la Calidad, de la versión ISO 9001:2008 hacia la versión ISO 9001:2015. Estado de México: Dirección General de Innovación. Fecha de consulta: 28 de mayo de 2019. Disponible en http://dgi.edomex.gob.mx/sites/dgi.edomex.gob.mx/files/files/GUIiADEAPOYOCORRECION.p df

Instituto Mexicano de Normalización y Certificación, A.C. (s.f.). Los 7 conceptos básicos de la nueva ISO 9001:2015. [Figura]. Consultado el 6 de mayo de 2019. Recuperado de http://www.amereiaf.mx/sites/default/files/legacy/congresos/cno2015_SLP/Los_7_Conceptos_B asicos_de_la_Nueva_ISO_9001-2015.pdf

Marquis, D. (1969). The anatomy of successful innovations. Innovation. 1(1), 35-48.

Organización Internacional de Normalización. (2015a). Sistemas de gestión de la calidad Fundamentos y vocabulario. Consultado el 4 de mayo 2019. Disponible en https://www.iso.org/obp/ui/\#iso:std:iso:9000:ed-4:v1:es

Organización Internacional de Normalización. (2015b). Brindando un nuevo brillo a la ISO 9001. Revista ISOfocus. No. 113.

Nava, V. M. y Jiménez, A. R. (2005). ISO 9000:2000 Estrategias para Implantar la Norma de Calidad para la Mejora Continua. México: Editorial Limusa.

Nava, V. M. (2006). ¿Qué es la Calidad? Conceptos, Gurús y Modelos Fundamentales. México: Editorial Limusa.

Martínez, F. (2007). Sistema de Gestión de la Calidad basado en ISO 9000:2000 enfocado en empresas de servicios. Tesis de Maestría. Universidad Veracruzana. México.

Miletić, L.Z., Jovanović, A.D., Jovanović, F.P., \& Buha, V.V. (2017). Designing priority databases for QMS according to ISO 9001: 2015 standard. Military Technical Courier. 65(3), 731-757.

Prudencio, G. (2016). Claves de la nueva Norma IATF 16949. AENOR. Revista de la Normalización y Certificación. 321, 30-35.

Retos en Supply Chain. (23 de Julio de 2014). Finalidad de las normas ISO: ¿para qué sirven? [Blog de EAE Business School]. Recuperado de https://retos-operaciones-logistica.eae.es/finalidad-delas-normas-iso-para-que-sirven/ 
La importancia de la información documentada. Caso de actualización documental de un Sistema de Gestión de la Calidad para certificación bajo la norma ISO 9001:2015

Rodríguez, M. (10 de Octubre de 2017). Historia de la norma ISO 9001. Evolución de la calidad ilustrada en la evolución de la ISO 9001. [Blog de Normas9000]. Recuperado de http://www.normas9000.com/Company_Blog/historia-iso-9001.aspx

Van Gigch, J. P. (2006). Teoría General de Sistemas. 3ra. Edición. México: Editorial Limusa. 